\title{
Study of air jet characteristics in air-conditioned rooms
}

\author{
Yulia R. Kareeva ${ }^{(a)}$, Vladimir N. Posokhin ${ }^{(a)}$, \\ Rinat G. Safiullin (a), \\ Ksenia A. Bliznyakova (a) \\ (a) Kazan State University of Architecture and Engineering \\ Kazan, 420043, Russian Federation, jkareeva2503@gmail.com
}

\begin{abstract}
The research deals with supply air jets in air-conditioned rooms which have different lengths. The study was conducted according to the numerical method using the Fluent software package. Discharge conditions are equal in all cases. As a result, dependences of main kinematic and geometric jet's characteristics (width and jet range, longitudinal velocity profiles, axial velocity, average velocity of the back flow, flow rates in the cross sections of the direct and back flows, distribution of static pressure along the length of the jet) on longitudinal constraint parameter are determined (length of room). The calculation results are presented in the form of corrections to the characteristics of a free jet, taking into account the influence of longitudinal constraint. It is found that influence of Archimede's buoyant force on the temperature distribution along the length of non-isothermal jet is inconsequential. Dependence of dimensionless temperature on the jet axis of dimensionless length of the room of the coordinate $\bar{x}$ is determined. The results can be used in calculation of airflow circulation in different purpose airconditioned rooms.
\end{abstract}

Keywords: non-isothermal jet, air-conditioned room, flow parameters, constraint parameter.

\section{INTRODUCTION}

Thermal comfort and air quality are the main goals of ventilation and air conditioning systems. Depending on the situation in the air-conditioned room, it is necessary to choose the appropriate air exchange scheme - the method of flows mixing or their driving out [1]. When choosing a mixing scheme, which has advantages for rooms with a high permissible velocity in the working area, air is conducted to the ceiling or floor, as well as horizontally, obliquely or vertically into the working area in the form of compact jets [2-6]. The flows created in the room using compact air supply are often non-isothermal and also carry various kinds of impurities. Thus, there is a task of calculating the velocity field, temperature and concentration in the jet and the room.

There are experimental and theoretical works which consider the characteristics of jets flowing into a room [7-12]. In recent years [10-12], when studying isothermal jets, it was found that the characteristics of the jet (width and 
jet range, longitudinal velocity profiles, axial velocity, average velocity of the back flow, flow rates in the cross sections of the direct and back flows, distribution of static pressure along the length of the jet) depend on the location and size of the holes through which air is supplied to and removed from the room, as well as on the size of the room itself.

Non-isothermal jets are studied much less, although it is obvious that the difference in the air densities of the flowing jet and the air-conditioned room can lead to significant deformation of the jet. The effect of non-isothermality on the distribution of jets in the rooms ventilated through active chilled beams was considered in the works $[13,14]$. In the works $[15,16]$, the characteristics of a nonisothermal jet in the cubage of large rooms are numerically studied by CFD modeling. However, there is no study of the jets in a confined space, when the dimensions of the jet and the room are commensurable, and confined air jets develop according to a dead end or flow pattern. Therefore, so far, we do not have satisfactory answers to such fundamentally important questions as energy-efficient air distribution in rooms, patterns of dynamic and thermal jets circulating in limited areas, etc.

\section{Materials and methods}

This article numerically determines the characteristics of a plane nonisothermal jet developing according to a dead-end scheme. Fig. 1 illustrates the pattern of the flow at the dead end, one end of which is muffled and the other is opened.

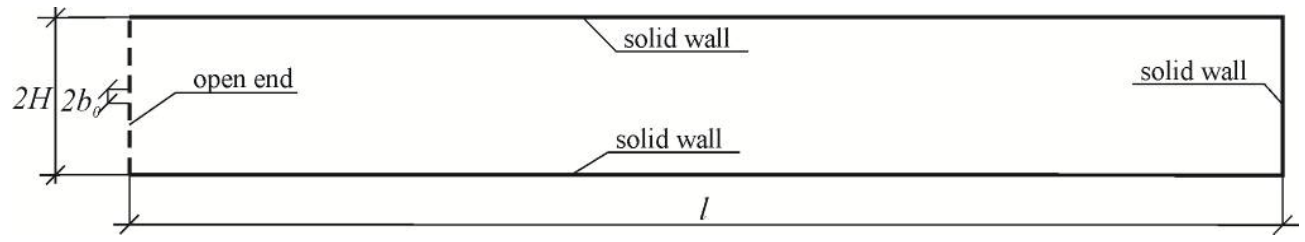

Figure 1. Configuration of the flow range

The study was conducted according to the numerical method using the Fluent software package on room models (dead ends) of the same height $2 H=0,34 \mathrm{~m}$, but different length: "long dead end" $l=2,52 m(l / H=14,82)$; "medium" dead end $l=0,82 m(l / H=4,82)$; "short" dead end $l=0,5 m(l / H=2,94)$. The conditions for the jet discharge are the same in all cases: the initial velocity $u_{0}=52,9 \mathrm{~m} / \mathrm{s}$; initial temperature $T_{0}=313 \mathrm{~K}$; width of the inflow slot $2 b_{0}=0,003 \mathrm{~m}$; initial flow rate per unit length of the slot $L_{0}=0,159 \mathrm{~m}^{2} / \mathrm{s}$. Low values $\Delta T_{0}=T_{0}-T_{\infty}=313-293=20 \mathrm{~K}$ and accordingly the criterion of Archimedes $A r_{0}=\frac{g b_{0}}{u_{0}^{2}} \cdot \frac{\Delta T_{0}}{T_{\infty}}=3,6 \cdot 10^{-7}$ are chosen so that the jet does not noticeably deform under the action of Archimede's buoyant force. 
Flow streamlines for all dead end lengths are shown in Fig. 2.

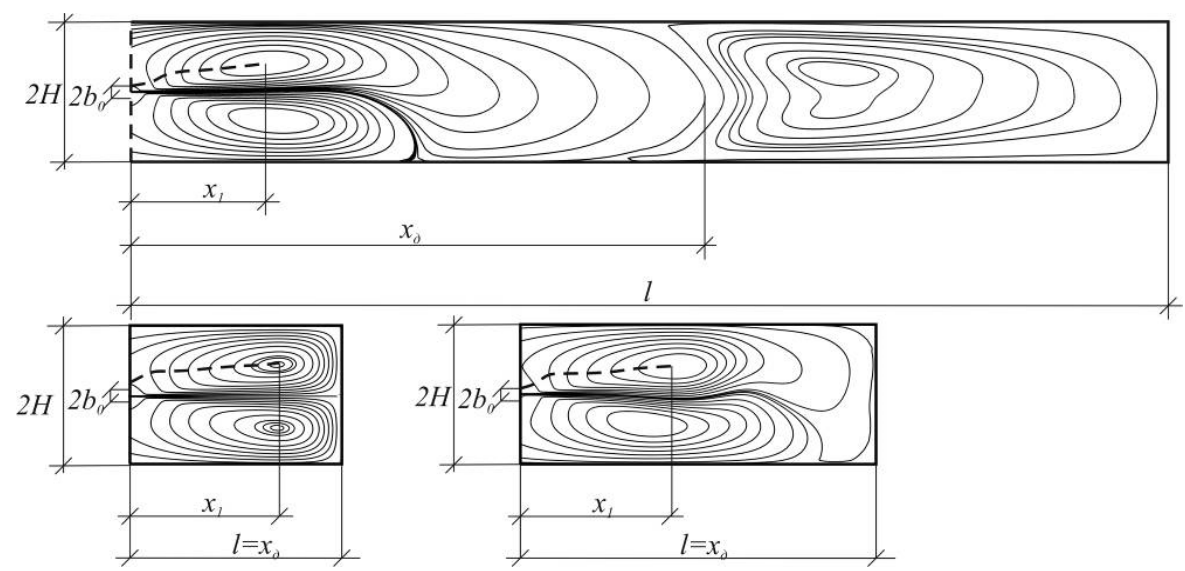

Figure 2. Flow streamlines

The calculation results are presented below in a dimensionless form. Relevant characteristics of the free jet are taken as scales, i.e.

$$
k_{u_{x}}=\frac{u_{x}^{c m}}{u_{x}^{c b}}, k_{T_{x}}=\frac{\Delta T_{x}^{c m}}{T_{x}^{c b}}, k_{y_{x}}=\frac{y_{x}^{c m}}{y_{x}^{c b}}, k_{L_{x}}=\frac{L_{x}^{c m}}{L_{x}^{c c}}, \bar{p}_{x}=\frac{2 p_{x}^{c m}}{\rho u_{0}^{2}} .
$$

The dead end height is taken as a linear scale, i.e. $\bar{x}=\frac{x}{H} ; \bar{b}_{0}=\frac{b_{0}}{H} ; \bar{l}=\frac{l}{H}$.

These are the necessary dependences for the free jet $[3,4]$ :

- axial velocity

$$
u_{x}^{c \beta}=2,62 \cdot \sqrt{\frac{T_{\infty}}{T_{0}}} \cdot \frac{u_{0} \sqrt{2 b_{0}}}{x}
$$

- axial excess temperature

$$
\Delta T_{x}^{c \beta}=2,52 \cdot \sqrt{\frac{T_{\infty}}{T_{0}}} \cdot \frac{\Delta T_{0} \sqrt{2 b_{0}}}{\sqrt{x}} ;
$$

- jet half-width

$$
y_{x}^{c B}=0,22 x
$$

- airflow rate in the jet

$$
L_{x}^{c \beta}=0,54 \cdot \sqrt{\frac{T_{\infty}}{T_{0}}} \cdot u_{0} \sqrt{2 b_{0}} \sqrt{x}
$$

It is emphasized that the formulas are suitable only for the main section of the jet. The lengths of the dynamic $x_{\mu \partial}$ and thermal $x_{H T}$ initial sections are approximately determined by the dependencies: 


$$
\begin{aligned}
& x_{\mu \partial}=6,86 \cdot \frac{T_{\infty}}{T_{0}} \cdot 2 b_{0}=0,019 ; \bar{x}_{н u}=0,11 ; \\
& x_{H T}=6,36 \cdot \frac{T_{\infty}}{T_{0}} \cdot 2 b_{0}=0,0178 ; \bar{x}_{H T}=0,105 .
\end{aligned}
$$

The results of numerical calculations are given below. Air ejection through the open end and the constraining effect of the back flow getting through the open end determine the complex behavior of the jet immediately after the discharge.

\section{Results and discussion}

Fig. 3 illustrates the change of the axial velocity in the jet, which complexly changes at $\bar{x}<2$. Further, the axial velocity drops dramatically to zero at $\bar{x}=\bar{x}_{\partial}$.

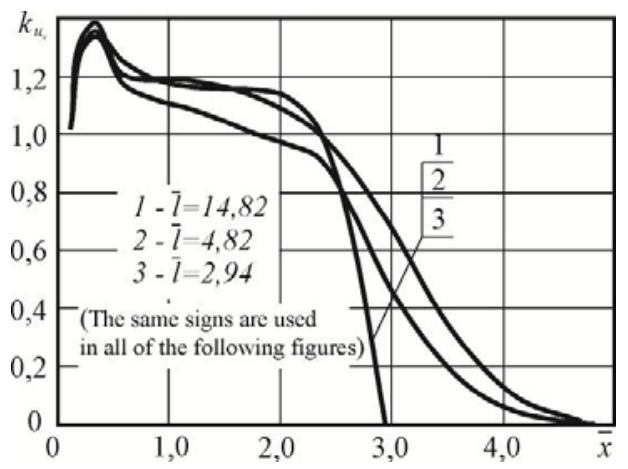

Figure 3. Change of the relative maximum velocity in the direct flow

It is important to note that the jet range in the "long" dead end, identified by Fig. 2 and Fig. 3 is not the same. The difference (approximately twofold) is due to the fact that the axial velocity at $\bar{x}>4,5-4,8$ is close to zero but not equal to zero. For practical purposes, it is more convenient to define the range as the distance at which the axial velocity reaches a certain low specified value.

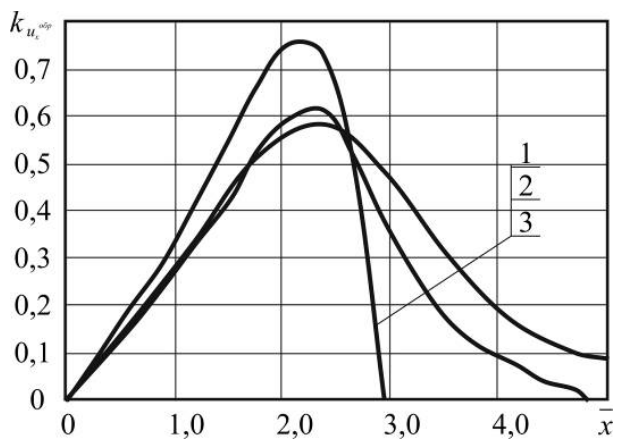

Figure 4. Change of the relative maximum velocity in the back flow 
Fig. 4 shows a change curve of relative maximum velocity in the back flow. Before the cross section $\bar{x} \approx 2 \quad k_{u_{\text {oб }}}$ increases to the maximum value.

Fig. 5 shows the longitudinal velocity profiles for the "long" dead end. The dashed line shows the velocity profiles made according to Tarnopolsky M.D. formula [8].
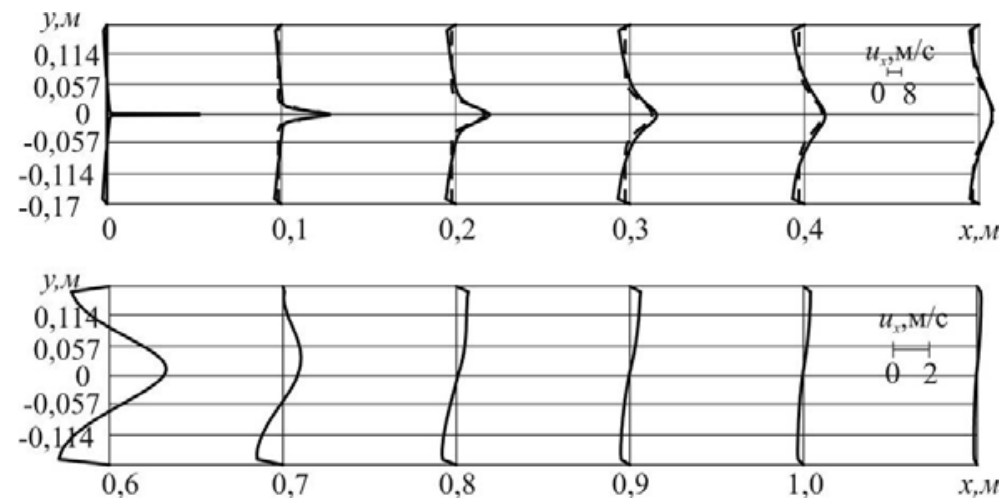

Figure 5. Profiles of the longitudinal velocity component

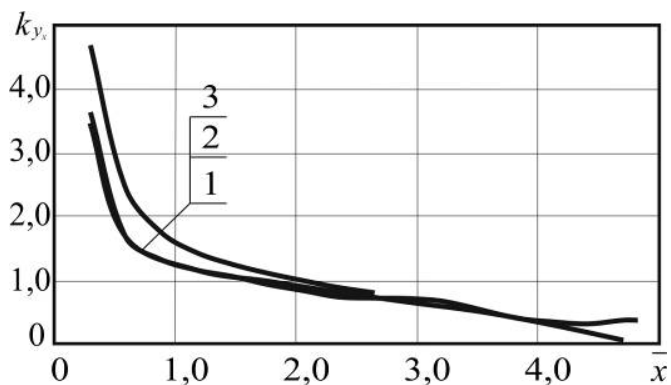

a)

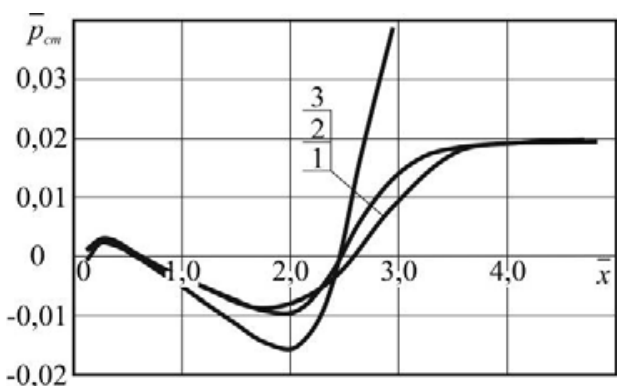

b)

Figure 6. Dependences for jet characteristics on the longitudinal constraint parameter $\bar{x}$ :

$\mathrm{a}$ - relative width of the jet in the direct and back flows; $\mathrm{b}$ - relative static pressure on the axis of the jet

Fig. 6a shows the results of calculations $k_{y}$. Immediately after the discharge, the width of the direct flow significantly exceeds the width of the free jet due to the air ejection through the open end $\left(k_{y_{x}}=3,5-4,5\right)$. Then, the jet width decreases actively, which is explained by rarefaction in this area (see Fig. 6 b). At $\bar{x} \approx 2$ rarefaction on the jet axis reaches a maximum in the same section $k_{y_{x}}=1$. This is followed by a turnaround area and the width of the direct flow decreases up to a minimum value at $\bar{x}=\bar{x}_{\partial}$. The static pressure is maximum here.

Fig. 7 shows the results of the calculation of the air flow in the direct stream. The ejection effect leads to the fact that at small distances the flow rate in 
the direct flow is noticeably greater than in the free jet, then the flow rate decreases to a minimum $\left(k_{L_{\mathrm{r}}} \approx 1,05\right)$ at $\bar{x} \approx 0,5$, then it increases slightly to a certain maximum in the cross section $\bar{x} \approx 2$. Further, the flow rate drops to almost zero at $\bar{x}=\bar{x}_{\partial}$.

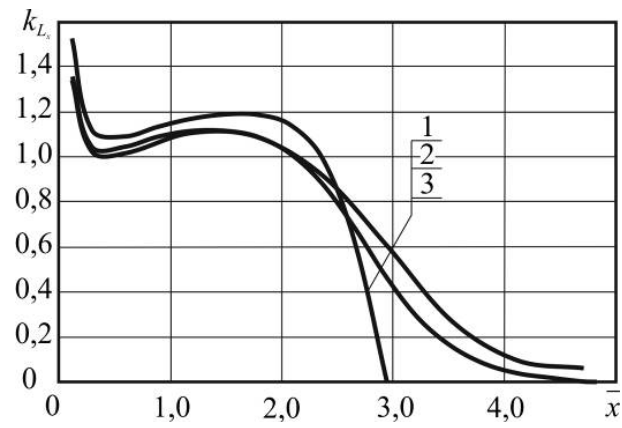

Figure 7. Change of the relative flow rate in the jet

Fig. 8a shows how the axial excess temperature varies along the length of the jet. Dead ends of different lengths are characterized by $\Delta T_{x}$ sharp drop at the distance $\bar{x}<0,2$, then $\Delta T_{x}$ practically does not change. The curve in the figure is plotted according to Tarnopolsky M.D. formula [8] for a free jet. It can be seen that the $\Delta T_{x}$ drop along the length of the free jet is noticeably more active.

The corresponding curves for the multiplier $k_{T}$ are shown in Figure 8b. At $\bar{x}<0,5$ there is also some deviation from the general pattern caused by the ejection effect. In the range of distances $0,5<\bar{x}<\bar{x}_{\partial}$, the dependence $k_{T_{x}}=k_{T_{x}}(x)$ is rather accurately approximated by the formula suitable for a dead end of any length

$$
k_{T_{x}}=0,001 \bar{x}^{3}-0,035 \bar{x}^{2}+0,64 \bar{x}+1,02
$$

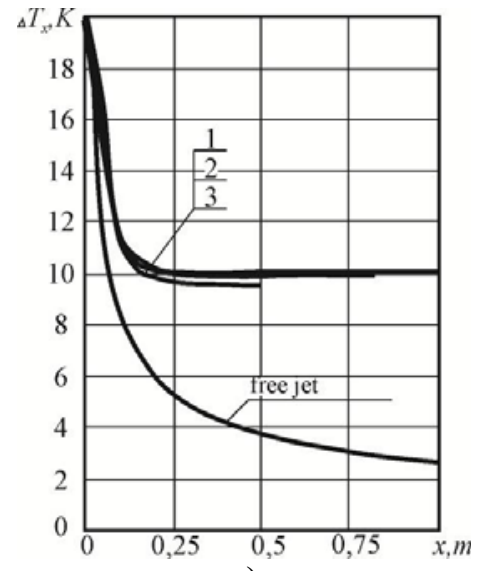

a)

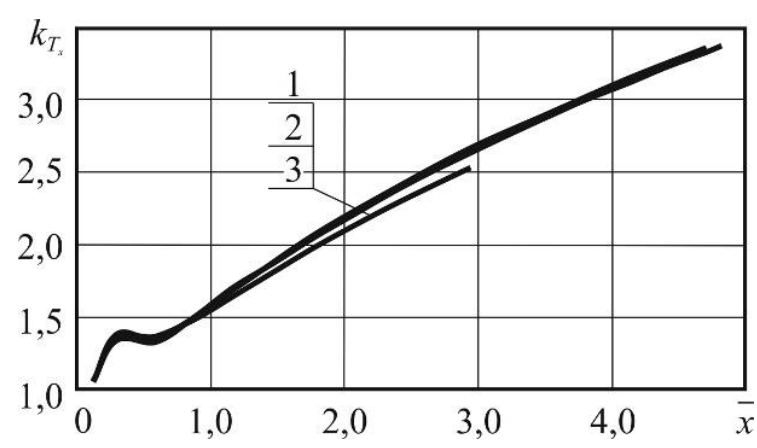

b)

Figure 8. Change of the excess temperature on the axis of the jet 
We can state the qualitative correspondence of the profiles calculated numerically and according to Tarnopolsky formula [8] at $x<0,5$. At large $x$ the profiles are asymmetric as they are in accordance with Figure 5.2.

Fig. 8 shows a change curve of the relative velocity in the direct (8a) and back flows (8b). As for axial velocity, before the start of the turnaround area $(\bar{x} \approx 2)$, the average velocity increases to the maximum value and then drops to 0 , indicating the range of the jet.
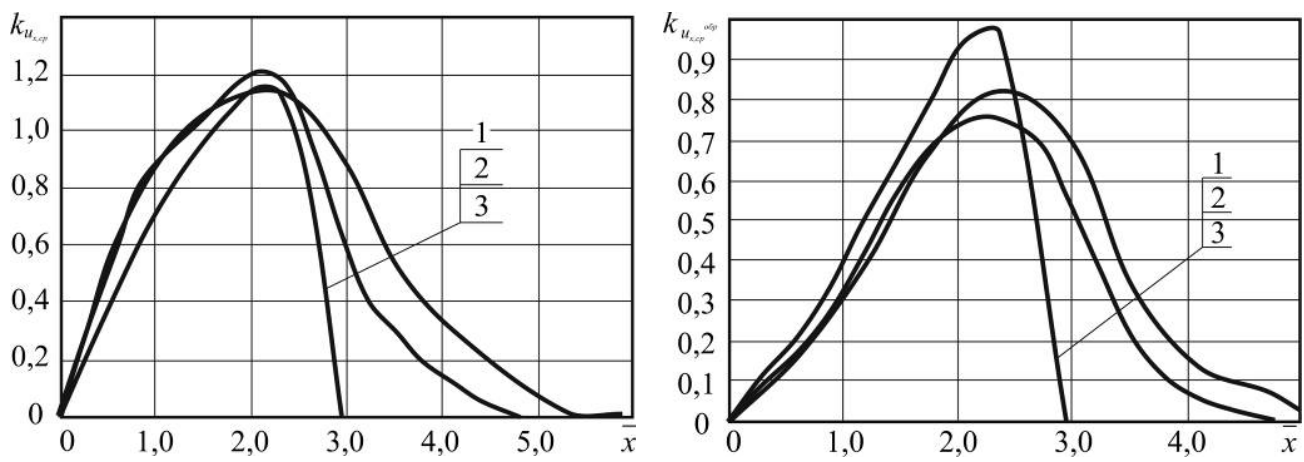

Figure 9. Change of the relative average velocity in direct and back flows

\section{CONCLUSION}

As a result of a numerical study, dimensionless dependences for the main characteristics of the jet on the longitudinal constraint parameter are obtained. Comparing the results obtained with the data given in the work [8], it can be said that the characteristics of the flow of the investigated jet are practically the same. This is explained by the fact that the jet is slightly non-isothermal and the longitudinal constraint factor affects only when $l \leq \bar{x}_{\partial}$. There is a dependence of the dimensionless temperature on the coordinate $\bar{x}$ suitable for air-conditioned rooms of any length.

\section{INSTRUMENTATION SYMBOLS}

$\begin{array}{ll}p & \text { pressure }(\mathrm{kPa}) \\ T & \text { temperature }(\mathrm{K})\end{array}$

$u \quad$ velocity $(\mathrm{m} / \mathrm{s})$

$L \quad \operatorname{rate}\left(\mathrm{m}^{3} / \mathrm{s}\right)$

\section{REFERENCES}

1. M. Cehlin, Doctorate Thesis, Stockholm (2006)

2. A. Sattari, Bengt. E. G. Fallenius, Jens H. M. Fransson, M. Sandberg, (Proceedings RoomVent - 12th International conference on air distribution in rooms, Trondheim, Norway, 2011)

3. I.A. Shepelev, Aerodynamics of air flows in the room (1978)

4. V.A. Bakharev, V.N. Troyanovsky (1958) 
5. G. Cao, J. Kurnitski, M. Ruponen, O. Seppänen, Applied Thermal Engineering, Elsevier, 29 (14-15) (2009)

6. P. Fillingham, I. Novosselov, AXiv preprint arXiv:1812.11220 (2018)

7. G. Abramovich, The theory of turbulent jets (1963)

8. M.D. Tarnopolsky The general movement of air in a ventilated room during jet flow (plane problem), Sat. Proceedings / Research Institute of Plumbing, Air conditioning, 18 (1966)

9. Y. Zou, Velocity Decay in Air Jets for HVAC Applications, ASHRAE Transactions 106, Part 2 (2000)

10. M. Zasimova, N. Ivanov (The eighth Polyakhov's reading: «Proceedings of the International Scientific Conference on Mechanics», 1959(1): 050033, 2018)

11. T-G. Malmstrom, Y. Zou (Roomvent Reading, UK, 2000).

12. G.I. Barenblatt, A.J. Chorin, V.M. Prostikishin, J. of Applied Mathematics, $102(2005)$

13. G. Cao, Kurnitski J., M. Ruponen, P. Mustakallio, O. Seppänen, Int. J. of Ventilation, Vol. 7, №4 (2009)

14. G. Cao, Kurnitski J., M. Ruponen, P. Mustakallio, O. Seppänen, Int. J. of Ventilation, Vol. 7, №2 (2008)

15. B-C.Wang, M. Koupriyanov, B. Tully, Building and Environment, 66 (2013)

16. K. Ito, K. Inthavong, T. Kurabuchi, T. Ueda, T. Endo, T. Omori, H. Ono, S. Kato, K. Sakai, Y. Suwa, H. Matsumoto, H. Yoshino, W. Zhang, J. Tu, Int. J. of Architectural Engineering Technology, 2 (2015) 